\title{
Exchange Rates and Markov Switching Dynamics
}

\author{
Yin-Wong Cheung \\ Economics Department, University of California, Santa Cruz, CA 96064 (cheung@ucsc.edu)
}

UIf G. ERLANDSSON

Economics Department, Lund University, SE 220 07, Sweden (ulf.erlandsson@nek.lu.se)

\begin{abstract}
This article presents a systematic and extensive empirical study on the presence of Markov switching dynamics in three dollar-based exchange rates. A Monte Carlo approach is adopted to circumvent the statistical inference problem inherent to the test of regime-switching behavior. Two data frequencies, two sample periods, and various specifications are considered. Quarterly data yield inconclusive evidence; the test rejects neither random walk nor Markov switching. Monthly data, on the other hand, offer unambiguous evidence of the presence of Markov switching dynamics. The results suggest that data frequency, in addition to sample size, is crucial for determining the number of regimes.
\end{abstract}

KEY WORDS: Exchange rate dynamics; Monte Carlo test; Regime switching; Sampling frequency.

\section{INTRODUCTION}

Since the beginning of the floating exchange rate era, there have been continuing efforts to explicate the behavior of exchange rates. However, most of these efforts appear to be futile. Many authors, including Cheung (1993), Cheung, Chinn, and Garcia Pascual (in press), Chinn and Meese (1995), Meese and Rose (1991), and Meese and Rogoff (1983), have demonstrated the inability of exchange rate models, both structural and time series models, to generate better forecasts than a naïve randomwalk specification.

Although a random-walk specification has garnered considerable empirical support, it has not dissuaded the profession from exploring predictable patterns in exchange rate movements and delineating possible interactions between exchange rates and their fundamentals. Among the recent attempts, Markov switching models appear to yield some encouraging results. Engel and Hamilton (1990), for instance, advocated using a Markov switching model that allows the exchange rate dynamics to alternate between regimes. These authors found that a two-regime Markov switching model performs well in both in-sample and out-of-sample periods for three exchange rate series. Engel (1994) extended the exercise to cover 18 exchange rate series and suggested that the Markov switching model yields superior direction of change forecasts. The successful use of Markov switching models to study exchange rate dynamics has also been reported by, for example, Bollen, Gray, and Whaley (2000) and Dewachter (2001). Marsh (2000), however, showed that Markov switching models for exchange rates are unstable over time and unsuitable for forecasting. Dacco and Satchell (1999) argued that the forecast performance of Markov switching models is very sensitive to misclassification of regimes.

The purpose of the exercise is to reevaluate the presence of Markov switching dynamics in exchange rate data. Most empirical studies on Markov switching dynamics do not formally test the number of regimes in the data. They assume, either explicitly or implicitly that data are drawn from a multiregime Markov switching process and estimate the model parameters accordingly. The absence of a formal test before estimation is probably due to the fact that the test for the number of regimes is a nonstandard statistical inference problem and the commonly used test statistics, including the likelihood ratio statistic, do not have their usual asymptotic distributions. However, without formal evidence of the existence of multiple regimes, estimation of Markov switching models may lead to spurious results. For instance, it is known that realizations of a random-walk process resemble observations displaying long swings. Fitting a Markov switching model to a (nearly) unit root process may generate spurious results and misidentify long swings due to unit root persistence as regime-switching behavior. Thus a formal test for the number of regimes is essential for determining the presence of Markov switching dynamics.

Besides statistical issues, the presence of regime-switching dynamics has important implications for theoretical models of exchange rate dynamics. For instance, Engel and Hamilton (1990, p. 689) pointed out that the apparent long swings in exchange rate data "pose important challenges for existing theory." Factors that may lead to regime-switching behavior include the "peso problem," the changing importance of chartists and fundamentalists in the foreign exchange market, differences between domestic and foreign monetary and fiscal policies, and the presence of transaction costs and diversity of opinions (De Grauwe and Vansteenkiste 2001; Engel and Hamilton 1990; Vigfusson 1996). The relevance of these factors depends critically on whether the observed exchange rates are generated from multiple regimes. Thus the research on exchange rate Markov switching dynamics has both important theoretical and statistical implications.

In this study we follow the lead of Rydén, Teräsvirta, and Åsbrink (1998) and adopt a Monte Carlo approach to test for the number of regimes in a Markov switching model. In particular, the data-specific empirical distribution of the likelihood ratio statistic is used to infer the presence (or absence) of regimeswitching behavior. Although simulation may not offer general conclusions, it circumvents the issues of nonstandard statistical inferences inherent in regime-switching modeling and provides

\footnotetext{
๑) 2005 American Statistical Association Journal of Business \& Economic Statistics July 2005, Vol. 23, No. 3 DOI 10.1198/073500104000000488
} 
some useful sample-specific results. Further, the use of dataspecific distributions helps mitigate finite-sample biases.

To anticipate the results, the Monte Carlo-based likelihood ratio test yields inconclusive evidence of Markov switching dynamics in the quarterly exchange rate data considered by Engel and Hamilton (1990). Although the test does not reject the random-walk specification, it provides no strong evidence for the two-regime Markov switching model. The ambiguous result is consistent with a number of possibilities. One possibility is that the data are not sufficiently informative to distinguish between the two specifications. To this end, we consider an extended quarterly sample and a monthly dataset. Even though the extended quarterly sample does not exhibit significant Markov switching dynamics, the monthly data do so. The results suggest that data frequency, in addition to sample length, is crucial for determining the number of regimes. The findings are robust to some variants of the Markov switching specification.

We must point out that this article is not a critique of a specific study on the Markov switching property of exchange rates. Rather, is seeks to provide a solid underpinning of the presence of Markov switching dynamics in exchange rate data.

The remainder of the article is organized as follows. Section 2 reports some preliminary analyses on the original Engel and Hamilton (1990) dataset. Section 3 describes the Monte Carlo-based test for the number of regimes, and reports test results from the quarterly dataset. Section 4 presents the results from analyzing monthly exchange rate data. Section 5 provides some concluding remarks.

\section{PRELIMINARY ANALYSES}

The first sample that we examined is the one used by Engel and Hamilton (1990). It contains quarterly dollar exchange rates of Deutsche mark, British pound, and French franc from 1973:IV to 1988:I, which were downloaded from ftp://weber.ucsd.edu/pub/jhamilto/markov2.zip. The randomwalk (with drift) specification is given by

$$
\Delta s_{t}=\mu+\varepsilon_{t},
$$

where $\Delta$ is the first-difference operator, $s_{t}$ is the log exchange rate at time $t, \mu$ is the drift term, and $\varepsilon_{t} \sim \mathrm{N}\left(0, \sigma^{2}\right)$ is an error term. The two-regime Markov switching model can be written as

$$
\Delta s_{t}=\sum_{i=1,2} I\left(S_{t}=i\right)\left[\mu_{i}+\varepsilon_{i t},\right],
$$

where $I(\cdot)$ is the indicator function, $\mu_{1} \neq \mu_{2}$ are the drift terms across regimes 1 and $2, \varepsilon_{i t} \sim \mathrm{N}\left(0, \sigma_{i}^{2}\right)$ is the regime-specific error term, and $S_{t}$ is the state (regime) variable. The state variable assumes a value of 1 or 2 and follows the transition probabilities $P_{i j}, i, j=1,2, \sum_{j=1,2} P_{i j}=1$ for $i=1,2$, where $P_{i j}$ is the probability of $S_{t}=j$ given $S_{t-1}=i$. (See, e.g., Hamilton 1989 for a discussion on the properties and estimation of a Markov regime switching model.) In the present study we use a fully unconstrained maximum likelihood method to estimate the model. The coefficient estimates of Markov switching models obtained from the fully unconstrained maximum likelihood method estimates are virtually the same as those originally reported by Engel and Hamilton (1990).
One critical and practical issue in estimating a Markov switching model is the possibility of multiple local maxima. In the current study, we used various randomized starting values in the optimization process to ensure that a global, instead of a local, maximum was obtained. Specifically, in the pretest stage, we generated artificial data from model specifications estimated from the Engel and Hamilton (1990) sample. We compared maximum likelihood values for each specification calculated from estimation process with 1-250 starting values. The starting values were random draws from normal distributions (for the mean parameter) and chi-squared distributions (for the variance parameter). Based on the simulation results, we determined that the choice of 100 starting values offers a reasonable trade-off between computing complexity and marginal gain in the maximum likelihood value. Thus 100 randomized starting values are used for each iteration in the estimation and Monte Carlo exercises reported in the subsequent sections.

It is commonly conceived that long swings are found in realizations from a random-walk process. Thus, before implementing the Monte Carlo-based test for the number of regimes, we conducted a pilot simulation study to examine what may be observed when a Markov switching model is fitted to a random-walk series. Specifically, we generated random-walk series according to parameters estimated from the British pound exchange rate data and fitted model (2) to the data. We found that the averages of transition probability estimates from these random-walk series were .89 , comparable to the transition probability estimates .93 and .91 obtained from the actual data. Obviously, the pilot study does not rule out the possibility of the existence of Markov switching dynamics in exchange rate data. However, the results underline the general conception that long swings in random-walk data may lead to spurious evidence of regime-switching behavior.

\section{TESTING FOR THE PRESENCE OF MARKOV SWITCHING DYNAMICS}

In general, the null and alternative hypotheses of the test for the presence of Markov switching can be stated as

$$
\mathrm{H}_{0} \text { : the number of regimes in the data is } N
$$

and

$$
\mathrm{H}_{1} \text { : the number of regimes in the data is } N+1 \text {. }
$$

For the current study, we consider mainly the $N=1$ case. That is, the data are drawn from a single regime under the null hypothesis and from two distinct regimes under the alternative. Let $\boldsymbol{\theta}_{N}$ and $\boldsymbol{\theta}_{N+1}$ be the parameter vectors under the null and alternative hypotheses and let $\hat{\boldsymbol{\theta}}_{N}$ and $\hat{\boldsymbol{\theta}}_{N+1}$ be the corresponding maximum likelihood estimators (MLEs). The likelihood ratio statistic is

$$
L R=2\left[L\left(\hat{\boldsymbol{\theta}}_{N+1}\right)-L\left(\hat{\boldsymbol{\theta}}_{N}\right)\right],
$$

where $L\left(\hat{\boldsymbol{\theta}}_{N+1}\right)$ and $L\left(\hat{\boldsymbol{\theta}}_{N}\right)$ are the log-likelihood functions evaluated at their respective MLEs, $\hat{\boldsymbol{\theta}}_{N+1}$ and $\hat{\boldsymbol{\theta}}_{N}$.

When testing for Markov switching, complexity arises because some parameters are not identified under the null. The presence of unidentified parameters invalidates the regularity conditions under which the likelihood ratio statistic can be 
shown to follow an asymptotic chi-squared distribution. If the null hypothesis is specified as $\mathrm{H}_{0}: \mu_{1}=\mu_{2}$ assuming $\sigma_{1}^{2} \neq \sigma_{2}^{2}$, then there is no problem of unidentified parameters, and a standard Wald test, for example, can be conducted. Indeed, Engel and Hamilton (1990) used the Wald test to investigate this null hypothesis. Nonetheless, the literature on testing for the presence of Markov switching dynamics typically considers the null hypothesis $\mathrm{H}_{0}: \mu_{1}=\mu_{2}$ and $\sigma_{1}^{2}=\sigma_{2}^{2}$. We conducted a small-scale simulation exercise to examine the finite-sample performance of the Wald test in this context. Specifically, we generated a random-walk series according to parameters estimated from the British pound data, fitted a Markov switching model to the simulated data, and used the Wald test to test the null hypothesis of $\mu_{1}=\mu_{2}$ assuming $\sigma_{1}^{2} \neq \sigma_{2}^{2}$. We repeated the exercise 100 times and found that the null hypothesis of $\mu_{1}=\mu_{2}$ was incorrectly rejected at the $5 \%$ level of significance in $75 \%$ of the cases. We speculate that both the nonlinear nature of the model and the apparent similarity between a randomwalk process and a Markov switching process contribute to the observed finite-sample performance of the Wald test. Thus the simulation results reenforce our impression that the Wald test approach is not suitable for the problem under investigation. Several procedures have been suggested to test for the number of regimes (see, e.g., Hansen 1992; Gong and Mariano 1997; Garcia 1998). These procedures derive the asymptotic behavior of the test statistic under some prespecific conditions and offer differing finite-sample performance for different model configurations. The Hansen (1992) test, for instance, involves a grid search over the parameter space and can be computationally complicated for a multiregime dynamic model. The Garcia (1998) and Gong and Mariano (1997) procedures are easier to implement, but not much is known about their small-sample performance. It should be noted that these procedures focus on testing between one or two regimes, and become quite complicated when a higher-dimension model is considered.

Following the lead of Rydén et al. (1998), we adopted a Monte Carlo approach to derive the empirical distribution of the likelihood ratio statistic, which we then used to determine the significance of the statistic computed from the actual data. For a given exchange rate series, we constructed the empirical distribution for testing $\mathrm{H}_{0}$ against $\mathrm{H}_{1}$ according to these steps: (a) Find the MLE $\hat{\boldsymbol{\theta}}_{N}$ that gives the best specification within the class of $N$-regime models; (b) use $\hat{\boldsymbol{\theta}}_{N}$ to generate a sample of the artificial data; (c) compute the likelihood statistic (3) from the generated data; and (d) repeat steps (b) and (c) $M$ times and store the simulated likelihood ratio statistics. We used the $M$ simulated likelihood ratio statistics to derive the empirical distribution to test the null. Specifically, the empirical $p$ value of the Markov switching test is given by $(m+1) /(M+1)$, where $m$ is the number of simulated statistics that are larger than the likelihood ratio statistic computed from the actual data series.

\subsection{Random Walk Against Markov Switching}

For the quarterly exchange rate data, (1) specifies the null hypothesis and (2) is the Markov switching model under the alternative. The sample likelihood ratio statistics are given in the second column of Table 1. The French franc and British
Table 1. Testing Random Walk Against Markov Switching, 1973:IV-1988:I

\begin{tabular}{lcccccccc}
\hline \hline & Sample & $p$ value & Mean & Median & SE & Skew & Max & Power \\
\hline DEM & 3.413 & .620 & 4.890 & 4.417 & 3.087 & .684 & 15.307 & .288 \\
GBP & 9.416 & .108 & 4.842 & 4.106 & 3.514 & 1.240 & 19.014 & .612 \\
FFR & 8.112 & .112 & 4.664 & 4.230 & 3.125 & 1.249 & 17.864 & .640 \\
\hline
\end{tabular}

NOTE: The likelihood ratio statistics computed from the exchange rate data (DEM, Deutsche mark; GBP, British pound; FFR, French franc) are reported under the column labeled "Sample." The $p$ values of these sample statistics derived from the empirical distributions are listed unde " $p$ value." The empirical distributions of the likelihood ratio statistic are generated from random walk models estimated from the data. Descriptive statistics of the empirical distributions are provided under "Mean," "Median," "SE," "Skew," and "Max." The column "Power" reports the frequency of rejections when these empirical distributions are used to evaluate the significance (at the $10 \%$ level) of the likelihood ratio statistics calculated from data generated from Markov switching processes estimated from exchange rate data.

pound series yielded similar sample likelihood ratio test statistics, which are larger than the one computed from the Deutsche mark data.

For each exchange rate series, we used the Monte Carlo approach to generate the sample specific empirical distribution of the likelihood ratio statistic. As stated earlier, we used 100 randomized starting values for each iteration in the numerical maximization algorithm. Due to computational complexity, we set the number of replications $M$ to 500 . For 500 replications and a $10 \%$ test used in the subsequent simulation, the $95 \%$ confidence interval of the rejection frequency is $10 \pm 2.6$ under the null hypothesis. Thus, even after taking sampling uncertainties into consideration, the inferences presented later are not affected. Some descriptive statistics of these empirical distributions and the associated $p$ values are given in Table 1. All the empirical distributions have their means larger than the medians. Furthermore, these are positively skewed distributions; that is, they have a long tail to the right. These descriptive statistics indicate that the empirical distributions are series-specific.

The empirical distributions of the likelihood ratio statistic cast doubts on the presence of Markov switching dynamics. According to the $p$ values, there is no strong evidence to reject the random-walk null in favor of the Markov switching model. Whereas the $p$ values for the French franc and British pound series are just over $10 \%$, the $p$ value for Deutsche mark data is above the $60 \%$ level and provides the weakest evidence against the random-walk hypothesis.

Are the nonrejection results driven by the low power of the testing procedure? To address the question on power, we applied the Monte Carlo-based test procedure to artificial data series generated according to the two-regime Markov switching models estimated from individual exchange rate series and tallied the likelihood ratio statistic. The rejection frequency under a $10 \%$ test (with critical values from the respective simulated null distribution) is reported as the empirical power in the last column of Table 1 . The power of the test against the Deutsche mark Markov switching specification is $28.8 \%$, which is quite low. Nonetheless, more than $60 \%$ of the simulated French franc and British pound Markov switching series are rejected at the $10 \%$ level. Note that there are fewer than 60 observations in this quarterly sample, the empirical power of the test seems reasonable, and the (low) power apparently is not an overwhelming concern for the nonrejection results.

The 1973:IV-1988:I sample period is arguably short. There may not be a sufficient number of distinct long swing cycles for 
Table 2. Testing Random Walk Against Markov Switching, 1973:IV-1998:IV

\begin{tabular}{lrccccccc}
\hline \hline & Sample & $p$ value & Mean & Median & SE & Skew & Max & Power \\
\hline DEM & 5.299 & .396 & 4.938 & 4.563 & 3.274 & .855 & 16.315 & .544 \\
GBP & 10.250 & .088 & 5.009 & 4.519 & 3.411 & .906 & 17.411 & .696 \\
FFR & 4.635 & .480 & 5.026 & 4.558 & 3.385 & .853 & 21.940 & .436
\end{tabular}

NOTE: The likelihood ratio statistics computed from the exchange rate data (DEM, Deutsche mark; GBP, British pound; FFR, French franc) are reported under the column labeled "Sample." The $p$ values of these sample statistics derived from the empirical distributions are listed under " $p$ value." The empirical distributions of the likelihood ratio statistic are generated from randomwalk models estimated from the data. Descriptive statistics of the empirical distributions are provided under "Mean," "Median," "SE," "Skew," and "Max." The column "Power" reports the frequency of rejections when these empirical distributions are used to evaluate the significance (at the $10 \%$ level) of the likelihood ratio statistics calculated from data generated from Markov switching processes estimated from exchange rate data.

the statistical procedure to determine the number of regimes Because of this concern, we considered the extended sample from 1973:I-1998:IV. The choice of ending the sample at 1998 is dictated by the introduction of the euro in 1999, which has fundamentally changed the dynamics of the dollar/mark and dollar/franc exchange rates. Simulation results based on parameter estimates from the extended sample are reported in Table 2. Despite of the fact that the extended sample has $75 \%$ more data points than the 1973:IV-1988:I sample, the test does not yield a definite result against the random-walk hypothesis for all three series. Compared with results in Table 1, the $p$ value for the Deutsche mark series declines to $39.6 \%$, but that of French franc increases to $48 \%$. The British pound series rejects the random-walk hypothesis at the $10 \%$ level but not at the 5\% level. The Monte Carlo-based test appears to attain a much higher level of power in the longer sample. The empirical power based on a $10 \%$ test ranges from $43.6 \%$ to $69.6 \%$. Thus far, there is no strong evidence against the random-walk null hypothesis.

\subsection{Markov Switching Against Random Walk}

One potential criticism of the results reported in the previous section is that the inferences are made with the random-walk specification as the null hypothesis. Although the power of the test does not appear to be a major concern, the setting does give the random-walk specification the benefit of the doubt. Specifically, the random-walk specification is rejected only if there is strong evidence against it. In this section we consider a heuristic approach to account for this asymmetric treatment.

A natural question to ask is "What is the behavior of the likelihood ratio statistic if the data are in fact generated from a Markov switching model?" If the Markov switching model gave the true data-generating process, then we would expect there to be substantial gain in the likelihood value between $L\left(\hat{\boldsymbol{\theta}}_{N+1}\right)$ and $L\left(\hat{\boldsymbol{\theta}}_{N}\right)$, and hence would expect the likelihood ratio statistic to be large. Alternatively, if a random-walk model generated the data, then the likelihood value would tend to be small. Heuristically speaking, when the likelihood ratio statistic computed from the actual data is small compared with the values of the likelihood ratio statistic generated from simulated Markov switching data, then it can be regarded as evidence against the Markov switching model and in favor of the random walk specification. Thus we simulated the data under a Markov switching specification and tallied the likelihood ratio statistics from these artificial data series. Then we evaluated
Table 3. Testing Markov Switching Against Random Walk, 1973:IV-1988:I

\begin{tabular}{lccrrrrrr}
\hline & Sample & $p$ value & Mean & Median & SE & Skew & Max & Power \\
\hline DEM & 3.413 & .152 & 7.505 & 6.228 & 4.752 & 1.739 & 37.427 & .316 \\
GBP & 9.416 & .380 & 12.371 & 11.128 & 7.057 & .991 & 41.389 & .528 \\
FFR & 8.112 & .344 & 12.030 & 10.942 & 6.928 & .663 & 32.483 & .444 \\
\hline
\end{tabular}

NOTE: The likelihood ratio statistics computed from the exchange rate data (DEM, Deutsche mark; GBP, British pound; FFR, French franc) are reported under the column labeled "Sample." The $p$ values of these sample statistics derived from the empirical distributions are listed under " $p$ value." The empirical distributions of the likelihood ratio statistic are generated from Markov switching models estimated from the data. Descriptive statistics of the empirical distributions are provided under "Mean," "Median," "SE," "Skew," and "Max." The column "Power" reports the frequency of rejections when these empirical distributions are used to evaluate the significance (at the $10 \%$ level) of the likelihood ratio statistics calculated from data generated from randomwalk processes estimated from exchange rate data.

whether the likelihood ratio statistic computed from exchange rate data is significantly smaller than the simulated likelihood ratios. The notion of the presence of Markov switching dynamics is rejected only if there is strong evidence against it - that is, the Markov switching model is rejected if only a very small portion of the simulated statistics is less than the likelihood ratio statistic from the actual data. In doing this, we reverse the asymmetric treatment built into the procedure considered in the previous subsection.

Table 3 reports the results of testing Markov switching dynamics against random walk in the 1973:IV-1988:I sample. We generated artificial data series according to Markov switching models fitted to the three exchange rate series. We constructed the likelihood ratio statistic (2) from each replication and tabulated then to derive the empirical distribution to determine the significance of the sample likelihood ratio statistic. The $p$ value is calculated as $m /(M+1)$, where $m$ is the number of simulated statistics smaller than the sample likelihood ratio statistic computed from actual data. Compared with Table 1, the descriptive statistics in Table 3 are consistent with the previous belief that the likelihood ratio statistic under the Markov switching model tends to be large. These empirical distributions are again positively skewed and data-specific.

The results, nevertheless, show that the sample likelihood ratio statistics are not too small compared with the simulated values. For the British pound and French franc series, the sample likelihood ratio statistics are larger than at least $30 \%$ of the statistics generated from simulated Markov switching data. The statistic for the Deutsche mark data is larger than $15 \%$ of the simulated statistics. Thus the results do not support the notion that the sample statistics are substantially less than what would be expected if data were generated via the Markov switching channel. Alternatively, we can say that there is no strong evidence to reject the Markov switching model in favor of the random-walk model. The power of the test, listed in the last column of Table 3, ranges from $32 \%$ to 53\%. That is, if the data are simulated with the random-walk specifications, then $32-53 \%$ of the simulated series give a likelihood ratio statistic smaller than the 10th percentile of the empirical distributions based on the Markov switching models. These power estimates are comparable to those in Table 1, in which a random walk is the null hypothesis.

The nonrejection of Markov switching result is enlightening. It underscores a fundamental issue in hypothesis testingnonrejection of the null hypothesis does not necessarily mean 
Table 4. Testing Markov Switching Against Random Walk, 1973:IV-1998:IV

\begin{tabular}{lrccrcccc}
\hline \hline & Sample & $p$ value & Mean & Median & SE & Skew & Max & Power \\
\hline DEM & 5.299 & .220 & 13.037 & 9.946 & 10.400 & 1.418 & 52.533 & .300 \\
GBP & 10.250 & .328 & 14.877 & 13.798 & 8.199 & .853 & 41.440 & .644 \\
FFR & 4.635 & .244 & 10.395 & 8.125 & 8.113 & 1.356 & 43.234 & .240
\end{tabular}

NOTE: The likelihood ratio statistics computed from the exchange rate data (DEM, Deutsche mark; GBP, British pound; FFR, French franc) are reported under the column labeled "Sample." The $p$ values of these sample statistics derived from the empirical distributions are listed under " $p$ value." The empirical distributions of the likelihood ratio statistic are generated from Markov switching models estimated from the data. Descriptive statistics of the empirical distributions are provided under "Mean," "Median," "SE," "Skew," and "Max." The column "Power" reports the frequency of rejections when these empirical distributions are used to evaluate the significance (at the $10 \%$ level) of the likelihood ratio statistics calculated from data generated from randomwalk processes estimated from exchange rate data.

that the null hypothesis is correct. In fact, what the results in Tables 1 and 3 tell us is that, given the parametric information retrieved from the data, we reject neither the random-walk nor the Markov switching specification. Table 4 contains the results for the 1973:IV-1998:IV sample. Even with this extended sample, the Monte Carlo results do not reveal sufficiently strong evidence to establish the random-walk model, although the test procedure does display reasonable empirical power (24-64\%) to reject Markov switching dynamics.

We conducted additional simulation experiments to investigate the power of the Monte Carlo-based test procedure (also see, e.g., Coe 2002). The sample size has a clear impact on the empirical power. Specifically, we used the random-walk and Markov switching specifications estimated from the Deutsche mark series to generate artificial data. These specifications gave the lowest power estimates in the exercise and thus would not inflate the power in the current simulation experiment. Performing a $10 \%$ test under the random-walk null hypothesis, the empirical power increased from $8 \%$ for a sample size of $50 \mathrm{ob}-$ servations to $77 \%$ for 300 observations and $96 \%$ for 500 observations. For the Markov switching null hypothesis and a $10 \%$ test, the empirical power improves from $24 \%$ for a sample size of 50 observations to $81 \%$ for 300 observations and $98 \%$ for 500 observations.

As shown by the simulation results, the test procedures have decent power to reject the respective hypotheses. Nonetheless, the Monte Carlo-based tests cannot offer a definite conclusion on whether there are Markov switching dynamics in the data. The data do not contain sufficient information to discriminate between the two alternatives, and the conclusion depends on which specification is being treated as the null hypothesis. Evidence of the presence or absence of Markov switching dynamics in the quarterly exchange data appears to be too strong.

\section{SAMPLING FREQUENCY}

It is conceived that the ability to detect regime-switching behavior depends on both the sample size and the frequency of observations. In the previous section we extended the sample to cover a 25-year time span and still could not confidently determine whether or not exchange rates have Markov switching dynamics. In this section we explore whether sampling frequency offers some useful information about Markov switching dynamics.
A higher sampling frequency can give better information on the dynamic property of exchange rate data. Suppose that an exchange rate switches between two regimes. If the time that the exchange rate is expected to stay in one regime is less than one quarter, then using quarterly data to test for Markov switching dynamics is deemed fruitless. Even if the expected duration is one quarter or a few quarters, quarterly samples may not offer sufficient observations within and across realized regimes to enable the test to disentangle the regime-switching behavior from the random-walk one. Relatively speaking, monthly observations have a better chance to retain and capture regimeswitching behavior. However, it is noted that the interpretation of the transition probabilities in the monthly two-states model differs from that in the quarterly two-states model. Suppose that the monthly data are generated from a two-states model and quarterly data are used. Each quarterly observation is from one of the two states. Giving a realization from, say, quarter $t$, there are four possible paths that lead to a state 1 observation at quarter $t+1$ and four possible paths that lead to a state 2 observation at $t+1$. Thus the transition probabilities are related but not the same under different data frequencies.

To explore the implication of data frequency, we examine month-end data of the same three exchange rates from 1973:10 1988:3 and 1973:10-1998:12. The two monthly samples are chosen to match the time spans of the two quarterly samples examined in the previous section. Results from these two periods should reveal the relative impact of sample size and sampling frequency on the test results. Before testing for the number of regimes, we checked for possible generalized autoregressive conditional heteroscedasticity $(\mathrm{GARCH})$ effects in the monthly data. There is no indication of GARCH effects in the Deutsche mark exchange rate data. The French franc and British pound data, on the other hand, have two local maximums in their likelihood functions; one maximum corresponds to a no-GARCH specification, and the other gives a nearly integrated GARCH model. Specifically, the no-GARCH specification gives the global maximum for the French franc series, and the GARCH specification gives the global maximum for the British pound series.

Because regime switching and structural breaks can be misidentified as a GARCH phenomenon (Cai 1994; Lamoureux and Lastrapes 1990; Mikosch and Stărică 2000), we modified the earlier Monte Carlo approach to determine whether a regime-switching model or a near-integrated GARCH model provides a better description for the data. The tests rejected the GARCH specification ( $p$ value $<.01$ ) but cannot reject the regime-switching specification $(p$ value $=87 \%$ ). Thus we proceeded to test for the number of regimes without GARCH dynamics.

The test results for the monthly data are summarized in Tables 5 and 6 . For the shorter sample, Table 5 provides some mixed evidence of Markov switching behavior. The randomwalk model is strongly rejected in the case of the French franc, marginally rejected in the cases of the British pound and the Deutsche mark. The empirical power of the test ranges from $39 \%$ to $74 \%$. On the other hand, there is no significant evidence against the Markov switching model.

For the 1973:10-1998:12 sample, the Monte Carlo-based test rejects the random-walk null hypothesis with $p$ values ranging 
Table 5. Test Results for the 1973:10-1988:3 Sample

\begin{tabular}{|c|c|c|c|c|c|c|c|c|}
\hline & Sample & $p$ value & Mean & Median & $S E$ & Skew & Max & Powe \\
\hline \multicolumn{9}{|c|}{ Random walk against Markov switching } \\
\hline & & & & & & & & \\
\hline & & & & & & & & \\
\hline & & .000 & & & & & & \\
\hline \multicolumn{9}{|c|}{ Markov switching against random walk } \\
\hline EM & & & & & 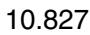 & & & \\
\hline & & & & & & & & \\
\hline FR & 25.289 & .460 & & & 46.1 & 1.515 & 246.347 & .26 \\
\hline
\end{tabular}

NOTE: The likelihood ratio statistics computed from the exchange rate data (DEM, Deutsch mark; GBP, British pound; FFR, French franc) are reported under the column labeled "Sample." The $p$ values of these sample statistics derived from the empirical distributions are listed under " $p$ value." Descriptive statistics of the empirical distributions are provided under "Mean," "Median," "SE," "Skew," and "Max."

In the first part of the table, the empirical distributions of the likelihood ratio statistic are generated from random-walk models estimated from the data. The column "Power" reports the frequency of rejections when these empirical distributions are used to evaluate the significance (at the $10 \%$ level) of the likelihood ratio statistics calculated from data generated from Markov switching processes estimated from exchange rate data.

In the second part, the empirical distributions of the likelihood ratio statistic are generated from Markov switching models estimated from the data. The column "Power" reports the frequency of rejections when these empirical distributions are used to evaluate the significance (at the $10 \%$ level) of the likelihood ratio statistics calculated from data generated from random-walk processes estimated from exchange rate data.

from .008 to .064 . When Markov switching is assumed to be the data-generating process, the sample likelihood ratio statistics are larger than $37-56 \%$ of the simulated statistics-indicating that the sample statistics are not likely to come from a randomwalk process. Further, the procedures have considerable power against the alternatives. The results in Table 6 provide strong evidence of exchange rates following Markov switching dynamics. In contrast to the quarterly data, the monthly data yield a much sharper inference on exchange rate dynamics.

One observation emerges pretty clearly in the quarterly and monthly results. Whereas an extended quarterly sample yields limited impact, an increase in sampling frequency has an appreciable effect on the test performance. However, the results from the quarterly sample 1973:IV-1988:I and the corresponding monthly sample 1973:10-1988:3 indicate that a mere change in data frequency may not suffice to deliver an unambiguous in-

Table 6. Test Results for the Sample 1973:10-1998:12

\begin{tabular}{|c|c|c|c|c|c|c|c|c|}
\hline & Sample & $p$ value & Mean & Median & $S E$ & Skew & Max & Pon \\
\hline \multicolumn{9}{|c|}{ Random walk against Markov switching } \\
\hline & & & & & & & & \\
\hline & & & & & & & & \\
\hline & & & & & & & & \\
\hline \multicolumn{9}{|c|}{ Markov switching against random walk } \\
\hline & & & & & & & & .42 \\
\hline & & & & & & & & \\
\hline & 22.888 & .504 & & & 38.151 & 1.615 & 181.319 & .24 \\
\hline
\end{tabular}

NOTE: The likelihood ratio statistics computed from the exchange rate data (DEM, Deutsche mark; GBP, British pound; FFR, French franc) are reported under the column labeled "Sample." The $p$ values of these sample statistics derived from the empirical distributions are listed under " $p$ value." Descriptive statistics of the empirical distributions are provided under "Mean," "Median," "SE," "Skew," and "Max."

In the first part of the table, the empirical distributions of the likelihood ratio statistic are generated from random-walk models estimated from the data. The column "Power" reports the frequency of rejections when these empirical distributions are used to evaluate the significance (at the $10 \%$ level) of the likelihood ratio statistics calculated from data generated from Markov switching processes estimated from exchange rate data.

In the second part, the empirical distributions of the likelihood ratio statistic are generated from Markov switching models estimated from the data. The column "Power" reports the frequency of rejections when these empirical distributions are used to evaluate the significance (at the $10 \%$ level) of the likelihood ratio statistics calculated from data generated from random-walk processes estimated from exchange rate data. ference about Markov switching dynamics. An increase in both sample size and sampling frequency, as represented by the extended monthly sample from 1973:10-1998:12, offers a better chance of detecting Markov switching dynamics in data.

Thus far it is assumed that under the Markov switching process, both the mean and the variance change across regimes at the same time. One alternative specification is to allow the mean and the variance to have their own switching dynamics. For instance, the mean $\mu_{i}$ is governed by a state variable $\mathrm{S}_{\mu t}=i$, and the variance $\sigma_{i}^{2}$ is governed by another state variable, $\mathrm{S}_{\sigma t}=i ; i=1,2$. In this case, the Markov switching model (2) can be rewritten as

$$
\Delta s_{t}=\sum_{i=1,4} I\left(S_{t}=i\right)\left[\mu_{i}+\varepsilon_{i t},\right]
$$

where $S_{t}$ is the state (regime) variable defined by $S_{t}=1$ when $S_{\mu t}=1$ and $S_{\sigma t}=1$, by $S_{t}=2$ when $S_{\mu t}=1$ and $S_{\sigma t}=2$, by $S_{t}=3$ when $S_{\mu t}=2$ and $S_{\sigma t}=1$, and by $S_{t}=4$ when $S_{\mu t}=2$ and $S_{\sigma t}=2$.

To check the robustness of our results, we re-did the Monte Carlo-based test for the 1973:10-1998:12 sample with (4) as the Markov switching alternative. The results are summarized in Table 7. The evidence is supportive of regime switching. The random-walk hypothesis is soundly rejected by the British pound and French franc data, but is only marginally rejected by the Deutsche mark series. On the other hand, there is no significant sign that the regime-switching model should be rejected. The nonrejection of Markov switching is probably not due to low power, because the power estimates are pretty high, in the range of $68-93 \%$. Thus the dynamics of exchange rate data are probably more complicated than described by the two-regime switching model given by (2). We also explored the possibility that there are three instead of two regimes in the monthly data. The results indicated that the Monte Carlo-based test does not have the ability to differentiate between the two- and threeregime specifications. To save space, we do not report the results here, but they are available from the authors on request.

Table 7. Extended Markov Switching or Random Walk 1973:10-1998:12

\begin{tabular}{|c|c|c|c|c|c|c|c|c|}
\hline & Sample & $p$ value & Mean & Median & $S E$ & Skew & Max & Power \\
\hline \multicolumn{9}{|c|}{ Random walk against Markov switching } \\
\hline & 15.009 & .109 & 8.120 & & 5.915 & 1.0 & & \\
\hline & 40.989 & 0 & & & & & & \\
\hline FFR & 25.227 & 0 & & & & & & \\
\hline \multicolumn{9}{|c|}{ larkov switching against random walk } \\
\hline$F M$ & & 25 & & & & & & 678 \\
\hline $\mathrm{BP}$ & & .4 & & & & 55 & & .724 \\
\hline FR & 25.227 & .300 & 32.152 & 32.292 & 14.161 & -.122 & 69.788 & .930 \\
\hline
\end{tabular}

NOTE: The likelihood ratio statistics computed from the exchange rate data (DEM, Deutsche mark; GBP, British pound; FFR, French franc) are reported under the column labeled "Sample." The $p$ values of these sample statistics derived from the empirical distributions are listed under " $p$ value." Descriptive statistics of the empirical distributions are provided under "Mean," "Median," "SE," "Skew," and "Max."

In the first part of the table, the empirical distributions of the likelihood ratio statistic are generated from random-walk models estimated from the data. The column "Power" reports the frequency of rejections when these empirical distributions are used to evaluate the significance (at the $10 \%$ level) of the likelihood ratio statistics calculated from data generated from extended Markov switching processes estimated from exchange rate data.

In the second part, the empirical distributions of the likelihood ratio statistic are generated from extended Markov switching models estimated from the data. The column "Power" reports the frequency of rejections when these empirical distributions are used to evaluate the significance (at the $10 \%$ level) of the likelihood ratio statistics calculated from data generated from randomwalk processes estimated from exchange rate data. 


\section{CONCLUSION}

This article has presented a systematic and extensive empirical study on the presence of Markov switching dynamics in three dollar-based exchange rates. Two data frequencies, two sample periods, and various specifications were considered. A Monte Carlo approach was adopted to circumvent the statistical inference problem inherent to the modeling of regime switching. Sample-specific empirical distributions were used to evaluate the significance of the sample likelihood statistic that tests the random-walk null hypothesis against the Markov switching alternative. The simulation results buttress the importance of using sample-specific distributions, because the behavior of the statistic is found to be contingent on the sample-specific dynamics. To address the asymmetry of having the random walk as the null hypothesis, we also examined the empirical behavior of the likelihood ratio statistic under the sample-specific Markov switching models. The use of samplespecific distributions also mitigates possible finite-sample biases.

The ability to discriminate between random walk and Markov switching depends on the power of the test and the information content of the data. Our simulation results show that the Monte Carlo-based test procedure has decent power against plausible data-specific alternatives. The quarterly and monthly results are consistent with the informational interpretation. Better information about Markov switching dynamics can be obtained by increasing the sample size and the sampling frequency. Our results indicate that increasing the sample size alone may not deliver the necessary information to disentangle regime-switching from random-walk dynamics. Quarterly exchange rate data of up to 24 years do not offer a clear-cut inference on the presence (or absence) of Markov switching dynamics. On the other hand, increasing the sampling frequency from quarterly to monthly delivers the necessary information to the data and allows the Monte Carlo-based test to extricate the Markov switching dynamics.

Overall, this article illustrates that the Monte Carlo-based test can be a promising procedure for detecting Markov switching dynamics. The result of testing for the presence of multiple regimes depends on the combination of sample size and sampling frequency. Our empirical evidence shows that for the monthly sample 1973-1998, there is strong evidence of Markov switching dynamics in exchange rate data.

\section{ACKNOWLEDGMENT}

The authors are very grateful to two anonymous referees for their insightful comments and useful suggestions. They also thank Michel Beine and participants at the Applied Econometric Association Meeting (2003) and the conference on Threshold Models and Recent Developments in Time Series (2004) for their comments and suggestions. Part of this work was done while Erlandsson was visiting the University of California, Santa Cruz (UCSC). Erlandsson thanks UCSC for its hospitality and the Royal Swedish Academy of Sciences and STINT for their financial support.

[Received June 2002. Revised May 2004.]

\section{REFERENCES}

Bollen, N. P. B., Gray, S. F., and Whaley, R. E. (2000), "Regime Switching in Foreign Exchange Rates: Evidence From Currency Option Prices," Journal of Econometrics, 94, 239-276.

Cai, J. (1994), “A Markov Model of Switching-Regime ARCH,” Journal of Business \& Economic Statistics, 12, 309-316.

Cheung, Y.-W., (1993), "Long Memory in Foreign Exchange Rates," Journal of Business \& Economic Statistics, 11, 93-102.

Cheung, Y.-W., Chinn, M., and Garcia Pascual, A. (in press), "Empirical Exchange Rate Models of the Nineties: Are Any Fit to Survive?" Journal of International Money and Finance, 24.

Chinn, M., and Meese, R. (1995), "Banking on Currency Forecasts: How Predictable Is Change in Money?" Journal of International Economics, 38, $161-178$.

Coe, P. (2002), "Power Issues When Testing the Markov Switching Model With the Sup Likelihood Ratio Test Using U.S. Output," Empirical Economics, 27, 395-401.

Dacco, R., and Satchell, S. (1999), "Why Do Regime-Switching Models Forecast so Badly?" Journal of Forecasting, 18, 1-16.

De Grauwe, P., and Vansteenkiste, I. (2001), "Exchange Rates and Fundamentals: A Non-Linear Relationship?" working paper, CEPR and University of Leuven.

Dewachter, H. (2001), "Can Markov Switching Models Replicate Chartist Profits in the Foreign Exchange Market?" Journal of International Money and Finance, 20, 25-41.

Engel, C. (1994), "Can the Markov Switching Model Forecast Exchange Rates?" Journal of International Economics, 36, 151-165.

Engel, C., and Hamilton, J. D. (1990), "Long Swings in the Dollar: Are They in the Data and Do Markets Know It?" American Economic Review, 80, 689-713.

Garcia, R. (1998), "Asymptotic Null Distribution of the Likelihood Ratio Test in Markov Switching Models," International Economic Review, 39, 763-788.

Gong, F., and Mariano, R. S. (1997), "Testing Under Non-Standard Conditions in Frequency Domain: With Applications to Markov Regime Switching Models of Exchange Rates and the Federal Funds Rate,' Staff Report 23, Federal Reserve Bank of New York.

Hamilton, J. D. (1989), "A New Approach to the Economic Analysis of Nonstationary Time Series and the Business Cycle," Econometrica, 57, 357-384.

Hansen, B. E. (1992), "The Likelihood Ratio Test Under Non-Standard Conditions: Testing the Markov Switching Model of GNP," Journal of Applied Econometrics, 7, S61-S82.

Lamoureux, C. G., and Lastrapes, W. D. (1990), "Persistence in Variance, Structural Change, and the GARCH Model," Journal of Business \& Economic Statistics, 8, 225-234.

Marsh, I. W. (2000), "High-Frequency Markov Switching Models in the Foreign Exchange Market,' Journal of Forecasting, 19, 123-134.

Meese, R., and Rogoff, K. (1983), "Empirical Exchange Rate Models of the Seventies: Do They Fit Out of Sample?" Journal of International Economics, 14, 3-24.

Meese, R., and Rose, A. K. (1991), “An Empirical Assessment of NonLinearities in Models of Exchange Rate Determination," Review of Economic Studies, 58, 603-619.

Mikosch, T., and Stărică, C. (2000), "Long-Range Dependence Effects and ARCH Modelling," technical report, Chalmers University.

Rydén, T., Teräsvirta, T., and Åsbrink, S. (1998), "Stylized Facts of Daily Return Series and the Hidden Markov Model," Journal of Applied Econometrics, 13, 217-244.

Vigfusson, R. (1996), "Switching Between Chartists and Fundamentalists: A Markov Regime-Switching Approach," Working Paper 96-101, Bank of Canada. 